\title{
Small ruminant pasteurellosis in Tigray region, Ethiopia: marked serotype diversity may affect vaccine efficacy
}

\author{
K. BERHE ${ }^{1} \dagger$, G. WELDESELASSIE ${ }^{2}$, J. BETTRIDGE ${ }^{3,4}$, R. M. CHRISTLEY ${ }^{3}$ AND \\ R. D. ABDI ${ }^{1,5 * \dagger}$ \\ ${ }^{1}$ Addis Ababa University, College of Veterinary Medicine and Agriculture, Department of Clinical Studies, \\ Bishoftu, Oromia, Ethiopia \\ ${ }_{2}^{2}$ Mekelle Regional Veterinary Diagnostic and Research Laboratory, Mekelle, Tigray, Ethiopia \\ ${ }^{3}$ Institute of Infection and Global Health, University of Liverpool, Leahurst Campus, Liverpool, UK \\ ${ }^{4}$ International Livestock Research Institute, Nairobi, Kenya \\ ${ }^{5}$ The University of Tennessee, Department of Animal Science, Knoxville, TN, USA
}

Received 18 August 2016; Final revision 23 November 2016; Accepted 17 December 2016; first published online 23 January 2017

\section{SUMMARY}

The aim of this study was to investigate the prevalent Bibersteinia, Mannheimia and Pasteurella serotypes, risk factors and degree of serotype co-infections in sheep and goats in the Tigray region of Ethiopia. Serum was collected from 384 sheep and goats from the Tanqua-Abergelle district of Tigray region using cross-sectional random sampling. An indirect haemagglutination test was used for serotyping. Risk factors for infections were evaluated by logistic regression. Potential clustering of multiple serotypes within individual animals due to common risk factors was evaluated by redundancy analysis. Eight serotypes were identified: all studied animals were serologically positive for at least one serotype. Overall, $355(92 \cdot 45 \%)$ of the animals were infected by four or more serotypes. Of the five risk factors studied, peasant association (PA), animal species, age (serotype A1), and bodyweight (serotype T15) were significantly associated with infection, but sex was not significant. Only PA explained a significant proportion of the variation (adjusted $R^{2}=0 \cdot 16$ ) in the serological responses. After the effect of PA was accounted for, T3 and T4; A7 and Pasteurella multocida A; and A7 and T10 were positively correlated for coinfection, while T4 and T10 were less likely to be found within the same animal. Diverse serotypes were circulating in the Tigray region and could be a challenge in selecting serotypes for vaccine.

Key words: Co-infection, B. trehalosi, M. haemolytica, P. multocida, serotype diversity.

\section{INTRODUCTION}

Mannheimia haemolytica and Pasteurella multocida have been identified as major economic problems in

\footnotetext{
* Author for correspondence: Dr R. Abdi, Addis Ababa University College of Veterinary Science, Bishoftu, Oromia - Clinical Studies, Hora Lake Road, Bishoftu, Oromia 34, Ethiopia.

(Email: retaduguma@gmail.com or rabdi@utk.edu)

$\dagger$ These authors contributed equally to this work.
}

ruminant production worldwide. P. multocida has 16 serotypes using lipopolysaccharide antigens as tested by a gel diffusion precipitation test [1] although it has five serogroups (A, B, D, E, F) using capsular antigens as tested by a passive haemagglutination test [2]. M. haemolytica is composed of a collection of 17 serotypes using capsular antigens as assayed by indirect haemagglutination [3] or co-agglutination test [4]. Globally, the 17 M. haemolytica serotypes 
were reorganized into Bibersteinia trehalosi containing four serotypes (T3, T4, T10, T15), M. haemolytica containing 12 serotypes (A1, A2, A5-A9, A12-A14, A16, A17) and M. glucosida with one serotype (A11) [5]. Despite the 17 serotypes, additional untypable isolates are recognized which account for more than $10-15 \%$ of the clinical isolates from ruminants [6].

Serotypes have demonstrated varying degrees of pathogenicity and virulence as assayed by clinical illness, post-mortem lung lesions and bacterial re-isolation rate [7]. Immunity is serotype specific and few [8] or no [4] serotypes cross-react. Distinct serotype associations with specific host species are noted and some serotypes have also distinct disease syndromes. Thus, P. multocida serotypes $\mathrm{B}$ and $\mathrm{E}$ cause haemorrhagic septicaemia of cattle, buffalo, goat, camel and deer; serotype D causes atrophic rhinitis of pigs and rabbits; serotypes A and D cause enzootic pneumonia and shipping fever of cattle, sheep and pigs; and serotypes $\mathrm{A}$ and $\mathrm{F}$ cause avian cholera of all bird species [9]. Similarly, M. haemolytica serotype A1 causes pneumonic pasteurellosis in cattle; M. haemolytica serotype A2 causes pneumonic pasteurellosis in sheep and goats and B. trehalosi serotypes cause septicaemic pasteurellosis in sheep and goats. Interspecies transmission of serotypes among domestic ruminants, as well as between domestic and wild ruminants, has been reported [10, 11], although some reports suggest that this is a rare epidemiological event [6].

Pasteurellosis and mannheimiosis are mainly influenced by a wide variety of environmental and management risk factors. Thus, the reduction or even elimination of such predisposing factors is of major importance. Antimicrobial drugs represent the most powerful tools to control such infections. However, increasing rates of antimicrobial resistance may markedly reduce the efficacy of the antimicrobial agents used to control Pasteurella and Mannheimia infections [12]. Vaccination is the best alternative practical control strategy to reduce the incidence and burden of the disease and to minimize antimicrobial use. Currently, several vaccine types exist against pasteurellosis globally [13]. Problems with vaccination arise where there is more than one serotype circulating, due to the lack of cross-protection [8].

At present, only a monovalent $P$. multocida serotype A vaccine is commercially available in Ethiopia [14], although P. multocida serotypes A and D [15] and 11 serotypes belonging to M. haemolytica [16] have long been detected. Consequently, repeated outbreaks are reported in Ethiopia [15, 17-19] even among vaccinated sheep and goats, which practitioners and communities ascribe to vaccine failure.

Serotypes have different levels of virulence, host-species adaptability with possible inter-species transmissibility, antigenicity, immunogenicity, drug resistance and a lack of inter-serotype cross-reactivity $[4,7]$. In Ethiopia, however, detailed information is lacking. Routine serotyping is not practised in Ethiopia due to the time and expense involved, and the lack of commercially available antisera, so that the serotypes of the circulating and outbreak-causing isolates remain unknown. Thus, the antigenicity, immunogenicity and cross-reactivity among the serotypes of the different eco-zones have not been studied. Therefore, this study was conducted to evaluate the (i) abundance, distribution and risk factors of infection for the different serotypes, and (ii) serotype mixed co-infections with potential clustering of Pasteurella, Mannheimia and Bibersteinia in sheep and goats in some selected areas of Tigray region.

\section{MATERIALS AND METHODS}

\section{Study area}

This study was conducted in five peasant associations (PAs), namely, Lemlem, Negede-Brhan, Gruwure, Mearey and Emba-Rufael of Tanqua-Abergelle district in Tigray regional state. A PA is the lowest administrative structure averaging about $50-80 \mathrm{~km}^{2}$ of land, with multiple PAs in each district. The study district is located $863 \mathrm{~km}$ north of Addis Ababa at $13^{\circ} 14^{\prime} 06^{\prime \prime} \mathrm{N}$ latitude, $38^{\circ} 58^{\prime} 50^{\prime \prime} \mathrm{E}$ longitude. It has a lowland agro-ecology with elevation between 938 and $2202 \mathrm{~m}$ a.s.1., daily maximum temperature range between $21^{\circ} \mathrm{C}$ and $31^{\circ} \mathrm{C}$ and annual rainfall between 400 and $650 \mathrm{~mm}$, the rainfall patterns are characterized as low and erratic. The production system in the district is extensive with traditional housing and grazing of natural pasture system. Mixed livestock farming is practised comprising of cattle, sheep, goats, mules, horses, and donkeys. Crops are grown mainly for their grains and to make use of crop residues for animal feed [20].

\section{Study animals, design and sample size}

Tanqua-Abergelle district was selected purposively due to its large population of sheep and goats, high reports of respiratory disease and pasteurellosis 
challenge and being the source of small ruminants for slaughter at a number of abattoirs in Tigray region, including the Abergelle export slaughterhouse of Mekelle. Abergelle sheep and goats, which were the focus of this study, have previously been characterized by household ownership, management practices, feed resource base, (re)productive performances, consumption and marketing [21]. Abergelle goats are a meat-type breed [22] although they can also produce milk [23].

A cross-sectional study design was employed. To the best of our knowledge, there have been no previous studies on pasteurellosis in the district. Thus, the sample size of study animals was calculated according to Thrusfield [24] using a 50\% expected prevalence and a $95 \%$ confidence interval $(Z=1.96)$ with a $5 \%$ desired absolute precision. The calculated sample size was 384 using the formula: $n=1 \cdot 96^{2} *(0 \cdot 5) *(1-$ $0 \cdot 5) / 0 \cdot 0025$. Of the $23 \mathrm{PAs}$ in the district, five PAs were selected randomly. Farmers who had sheep and goats were selected from registers of PA members using simple random sampling and all animals belonging to selected farmers were included in the study. Selection of additional participants continued until the calculated number of ruminants was achieved. The name of the PA and the owner of the small ruminants, as well as species, age, sex and bodyweight of each study animal were recorded during the survey.

\section{Sample collection, transportation and laboratory analysis}

From each animal $10 \mathrm{ml}$ blood was collected aseptically from the jugular vein into a plain tube. The blood was allowed to clot without shaking for $2 \mathrm{~h}$ at room temperature, and stored horizontally overnight at $4{ }^{\circ} \mathrm{C}$. The serum was then separated from the clot by centrifugation at $3000 \mathrm{rpm}$ for $10 \mathrm{~min}$ and transferred to cryovials, labelled and transported in an icebox to the district veterinary clinic and kept frozen $\left(-20^{\circ} \mathrm{C}\right)$ until analysis. The collected sera were transported on ice to the National Veterinary Institute, Bishoftu, where they were tested using an indirect haemagglutination (IHA) test as described by Biberstein et al. [3] and Biberstein \& Thompson [25]. An agglutination rate of $>50 \%$ was taken as positive [26]. Positive agglutination was confirmed by the presence of a dense clot at the bottom of the microtitre well and negative result by the presence of uniform suspension of the red blood cells. Each sample was tested for eight different serotypes, namely, M. haemolytica types A1,
A2, A7, P. multocida type A and B. trehalosi types T3, T4, T10 and T15. Reference serotypes were obtained from CIRAD-EMVT, France.

\section{Data analysis}

Microsoft Excel 2007 (Microsoft Corp., USA) was used for data management. Descriptive statistics were used to summarize the data. PA, age, sex, species and bodyweight of the animals were considered as potential risk factors for Pasteurella, Mannheimia and Bibersteinia seropositivity. Associations with the dependent variable (seropositivity) were assessed initially using cross-tabulation and univariable logistic regression using SPSS software v. 20 (IBM Corp., USA) to compute the odds ratio associated with potential risk factors. Non-collinear variables that presented a $P$ value $\leqslant 0 \cdot 25$ in the univariable analyses were included in the multivariable logistic regression model. In the final model, results were considered significant at $P<0.05$.

\section{Multivariable analysis}

To identify clustering of seropositivity to multiple serotypes within individual animals it was necessary to allow for potential clustering of seropositivity due to common risk factors, as some PAs, and also the species of the animals were risk factors for more than one serotype. We used redundancy analysis (RDA), a form of multivariable analysis that combines a principal component analysis, to identify clusters, with regression to identify significant explanatory variables, in order to address this question. RDA was performed using R software v. 3·0·2 (R Core Team 2013), using the $\mathrm{R}$ package 'vegan' [27], according to the methods described by Borcard et al. [28]. The binary data for each of the eight Mannheimia, Pasteurella and Bibersteinia strains was used to construct a distance matrix of responses, which was transformed using a Hellinger transformation, to reduce the impact of double negatives in the dataset, as described by Borcard et al. [28]. The categorical explanatory variables considered were PA, age, sex, species and bodyweight. A forward selection process was used to select significant variables which explained the greatest proportion of variance in the response data, and permutation tests used to test significance of RDA axes. Triplots were produced according to correlations between variables (scaling 2 in the vegan package). 


\section{RESULTS}

\section{Univariable analysis of serotype distribution in sheep and goats in different PAs}

Of 384 sheep and goats studied, $98 \cdot 7 \%, 66 \cdot 9 \%$ and $98 \cdot 7 \%$ were seropositive to $M$. haemolytica, $P$. multocida serotype A and B. trehalosi, respectively. In total, eight serotypes were detected in the study area. Higher prevalence of serotypes A1, A2, T3, T4 and T15 were observed in goats than in sheep; this association was statistically significant $(P<0 \cdot 05)$ for all serotypes except T15. Serotypes A7 and T10 were more prevalent in sheep, as was $P$. multocida serotype A, overall, although this latter finding was not statistically significant (Table 1). For two serotypes (A1 and T15) prevalence did not differ significantly between the five PAs, although all other serotypes showed statistically significant $(P<0.05)$ variation compared to the reference PA (Lemlem). In Mearey, Negede-Brhan and Emba-Rufael, A2, T3 and T4 prevalence was lower; while prevalence of A7, M. haemolytica and T10 (except in Emba-Rufael) were significantly higher, compared to Lemlem. In Gruwure, only T4 prevalence was significantly lower, while A7 and M. haemolytica prevalence were higher (Table 1).

\section{Univariable analysis of serotype distribution in small ruminants of different age, bodyweight and sex}

Age of the animals was not associated with seven of the serotypes studied, the exception was $M$. haemolytica serotype A1 which was significantly more prevalent $(P<0.05)$ in age groups $>2.5$ years compared to the reference age group ( $<1$ year). Only $B$. trehalosi T15 showed a statistically significant association with bodyweight, being more prevalent in animals weighing 11-20 kg compared to the reference bodyweight group $(1-10 \mathrm{~kg})(P<0 \cdot 05)$. Sex of the animals did not have any significant $(P>0.05)$ association with any of the eight serotypes studied (Table 2).

\section{Multivariable logistic regression for evaluation of risk factors in the final model}

A multivariable logistic regression identified that only PA (location) and species were significant risk factors for the majority of the serotypes. M. haemolytica (serotypes A2 and A7), and B. trehalosi (serotypes T3, T4 and T10) infections were significantly associated with particular PAs. However, some serotypes and PAs had no association: $M$. haemolytica serotype A2 vs. Negede-Brhan; B. trehalosi serotypes T3, T4 and T10 vs. Gruwure; and B. trehalosi serotype T10 vs. Negede-Brhan. After controlling for PA, goats had increased odds of B. trehalosi serotype T3 and T4 seropositivity (Table 3 ).

\section{RDA for evaluation of potential clustering and distribution of serotype mixed co-infections in sheep and goats}

There was no single animal that was seronegative for all pathogens tested. Of 384 animals, $11 \cdot 98 \%, 23 \cdot 70 \%$, $25 \cdot 26 \%, 22 \cdot 66 \%$ and $8 \cdot 85 \%$ were seropositive to four, five, six, seven and eight different combinations of serotypes, respectively. That is, $355(92.45 \%)$ animals were infected by four or more serotype combinations. A total of 103 different serotype combination patterns were observed, which are summarized in Table 4 and a detailed distribution listed in Table 5. The most prevalent co-positive pattern involved all serotypes except T10, and was found in 43/384 (11.2\%) animals. Positivity to all eight serotypes was the next most frequent finding (34/384; $8 \cdot 85 \%)$, and 30/384 (7.81\%) were co-positive to A1A2T3T4T15. There were 56 patterns that were only observed in a single animal (Table 5).

Subsequently, the level of within-animal clustering (co-positivity) of serotypes and risk factors affecting clustering tendencies were evaluated using RDA (Fig. 1). Only PA explained a significant proportion of the variation (adjusted $R^{2}=0 \cdot 16$ ) in the serological responses. After this risk factor was accounted for, B. trehalosi serotypes $\mathrm{T} 3$ and $\mathrm{T} 4$ were observed to be positively correlated within animals; however, T4 serotype was observed to have negative correlations with the T10 strain. M. haemolytica strain A7 was clustered with $P$. multocida serotype A, and to a lesser extent with $B$. trehalosi serotype T10 within animals.

\section{DISCUSSION}

Pasteurellosis is a pneumonic or acute systemic disease of small ruminants, with the precise syndrome determined by specific serotypes. In this regard pasteurellosis is a significant problem in extensive freeroaming smallholder farming conditions as animals are exposed to a wide spectrum of stressful conditions [29, 30]. Serotyping remains an important step in delineating $P$. multocida, B. trehalosi and M. haemolytica isolates, as antigens for vaccines are still based on capsular types and cross-reaction is absent in different serotypes [4]. In this study, the abundance, 
Table 1. M. haemolytica, P. multocida and B. trehalosi serotype prevalence and the associated risk factors in sheep and goats in different peasant associations of Tigray region

\begin{tabular}{|c|c|c|c|c|c|c|c|c|c|c|c|}
\hline Variables & $\begin{array}{l}\text { Summary } \\
\text { statistics }\end{array}$ & A1 & $\mathrm{A} 2$ & A7 & Total A & MA & $\mathrm{T} 3$ & $\mathrm{~T} 4$ & $\mathrm{~T} 10$ & $\mathrm{~T} 15$ & Total T \\
\hline Sheep $(n=192)$ & Positive (\%) & $144(75 \cdot 0)$ & $132(68 \cdot 8)$ & $139(72 \cdot 8)$ & $187(97 \cdot 4)$ & $134(69 \cdot 8)$ & $149(77 \cdot 6)$ & $99(51 \cdot 6)$ & $90(46 \cdot 9)$ & $167(87 \cdot 0)$ & $189(98 \cdot 4)$ \\
\hline Goats $(n=192)$ & Positive (\%) & $161(83 \cdot 9)$ & $151(78 \cdot 6)$ & $112(58 \cdot 3)$ & $189(98 \cdot 4)$ & $123(64 \cdot 1)$ & $175(91 \cdot 8)$ & $141(73 \cdot 4)$ & $69(35 \cdot 9)$ & $177(92 \cdot 2)$ & $190(99 \cdot 0)$ \\
\hline Total $(n=384)$ & Positive (\%) & $305(79 \cdot 4)$ & $283(73 \cdot 7)$ & $251(65 \cdot 5)$ & $379(98 \cdot 7)$ & $257(66 \cdot 9)$ & $324(84 \cdot 4)$ & $240(62 \cdot 5)$ & $159(41 \cdot 4)$ & $344(89 \cdot 6)$ & $379(98 \cdot 7)$ \\
\hline Sheep & & Ref. & Ref. & Ref. & Ref. & Ref. & Ref. & Ref. & Ref. & Ref. & Ref. \\
\hline \multirow[t]{2}{*}{ Goats } & OR $(95 \% \mathrm{CI})$ & $\begin{array}{l}1 \cdot 73 \\
(1 \cdot 04-2 \cdot 86)\end{array}$ & $\begin{array}{l}1 \cdot 67 \\
(1 \cdot 05-2 \cdot 65)\end{array}$ & $\begin{array}{l}0 \cdot 52 \\
(0 \cdot 34-0 \cdot 80)\end{array}$ & $\begin{array}{l}1 \cdot 67 \\
(1 \cdot 05-2 \cdot 65)\end{array}$ & $\begin{array}{l}0 \cdot 77 \\
(0 \cdot 50-1 \cdot 18)\end{array}$ & $\begin{array}{l}2 \cdot 97 \\
(1 \cdot 63-5 \cdot 43)\end{array}$ & $\begin{array}{l}2 \cdot 59 \\
(1 \cdot 69-3 \cdot 98)\end{array}$ & $\begin{array}{l}0 \cdot 64 \\
(0 \cdot 42-0 \cdot 96)\end{array}$ & $\begin{array}{l}1 \cdot 77 \\
(0 \cdot 90-3 \cdot 46)\end{array}$ & $\begin{array}{l}1 \cdot 51 \\
(0 \cdot 25-9 \cdot 12)\end{array}$ \\
\hline & $P$ value & 0.033 & $0 \cdot 0284$ & 0.0031 & 0.0284 & $0 \cdot 2333$ & $0 \cdot 0004$ & $<0.001$ & 0.03 & 0.098 & 0.6548 \\
\hline Lemlem $(n=80)$ & Positive (\%) & $63(78 \cdot 8)$ & $65(81 \cdot 3)$ & $80(10 \cdot 1)$ & $75(93.8)$ & $25(31 \cdot 3)$ & $78(97 \cdot 5)$ & $67(83.8)$ & $11(13 \cdot 8)$ & $71(88 \cdot 8)$ & $80(100 \cdot 0)$ \\
\hline $\begin{array}{l}\text { Negede-Brhan } \\
(n=80)\end{array}$ & Positive (\%) & $62(77 \cdot 5)$ & $54(67 \cdot 5)$ & $64(80 \cdot 0)$ & $79(98 \cdot 8)$ & $71(88 \cdot 8)$ & $60(75 \cdot 0)$ & $47(58 \cdot 8)$ & $55(68 \cdot 8)$ & $76(95 \cdot 0)$ & $79(98 \cdot 8)$ \\
\hline Gruwure $(n=82)$ & Positive (\%) & $67(81 \cdot 7)$ & $75(91 \cdot 5)$ & $62(75 \cdot 6)$ & $81(98 \cdot 8)$ & $60(73 \cdot 2)$ & $75(91 \cdot 5)$ & $57(69 \cdot 5)$ & $19(23 \cdot 2)$ & 77 (93.9) & $81(98 \cdot 8)$ \\
\hline Mearey $(n=76)$ & Positive (\%) & $61(80 \cdot 3)$ & $50(65 \cdot 8)$ & $63(82 \cdot 9)$ & $76(100 \cdot 0)$ & $57(75 \cdot 0)$ & $56(73 \cdot 7)$ & $22(28.9)$ & $56(73 \cdot 7)$ & $59(77 \cdot 6)$ & $73(96 \cdot 1)$ \\
\hline $\begin{array}{l}\text { Emba-Rufael } \\
\quad(n=66)\end{array}$ & Positive (\%) & $52(78 \cdot 8)$ & $39(59 \cdot 1)$ & $54(81 \cdot 8)$ & $65(98 \cdot 5)$ & $44(66 \cdot 7)$ & $55(83 \cdot 3)$ & $47(71 \cdot 2)$ & $18(27 \cdot 0)$ & $61(92 \cdot 4)$ & $66(100 \cdot 0)$ \\
\hline Lemlem & & Ref. & Ref. & Ref. & & Ref. & Ref. & Ref. & Ref. & Ref. & \\
\hline \multirow[t]{2}{*}{ Negede-Brhan* } & OR $(95 \% \mathrm{CI})$ & $\begin{array}{l}0.92 \\
(0.43-1.96)\end{array}$ & $\begin{array}{l}0 \cdot 47 \\
(0 \cdot 23-0 \cdot 99)\end{array}$ & $\begin{array}{l}35 \cdot 5 \\
(14 \cdot 24-88 \cdot 48)\end{array}$ & - & $\begin{array}{l}17 \cdot 35 \\
(7 \cdot 49-40 \cdot 16)\end{array}$ & $\begin{array}{l}0 \cdot 07 \\
(0 \cdot 01-0 \cdot 34)\end{array}$ & $\begin{array}{l}0 \cdot 27 \\
(0 \cdot 13-0 \cdot 58)\end{array}$ & $\begin{array}{l}13 \cdot 8 \\
(6 \cdot 24-0 \cdot 48)\end{array}$ & $\begin{array}{l}2 \cdot 40 \\
(0 \cdot 71-8 \cdot 16)\end{array}$ & - \\
\hline & $P$ value & $0 \cdot 8484$ & 0.0486 & $<0 \cdot 001$ & - & $<0 \cdot 001$ & $<0.001$ & $<0.001$ & $<0.001$ & $0 \cdot 1584$ & - \\
\hline \multirow[t]{2}{*}{ Gruwure* } & OR $(95 \% \mathrm{CI})$ & $\begin{array}{l}1 \cdot 2 \\
(0 \cdot 55-\cdot 61)\end{array}$ & $\begin{array}{l}2 \cdot 47 \\
(0 \cdot 9-6 \cdot 43)\end{array}$ & $\begin{array}{l}27 \cdot 5111 \cdot 32- \\
66 \cdot 84)\end{array}$ & - & $\begin{array}{l}6 \cdot 00 \\
(3 \cdot 04-11 \cdot 84)\end{array}$ & $\begin{array}{l}0 \cdot 27 \\
(0 \cdot 05-1 \cdot 36)\end{array}$ & $\begin{array}{l}0 \cdot 44 \\
(0 \cdot 20-0 \cdot 94)\end{array}$ & $\begin{array}{l}1 \cdot 89 \\
(0 \cdot 83-4 \cdot 28)\end{array}$ & $\begin{array}{l}1 \cdot 95 \\
(0 \cdot 62-6 \cdot 10)\end{array}$ & - \\
\hline & $P$ value & 0.63 & 0.0635 & $<0 \cdot 001$ & - & $<0 \cdot 001$ & $0 \cdot 1141$ & 0.0348 & $0 \cdot 1263$ & $0 \cdot 2500$ & - \\
\hline \multirow[t]{2}{*}{ Mearey* } & OR $(95 \% \mathrm{CI})$ & $\begin{array}{l}1 \cdot 09 \\
(0 \cdot 50-2 \cdot 39)\end{array}$ & $\begin{array}{l}0 \cdot 44 \\
(0 \cdot 21-0 \cdot 92)\end{array}$ & $\begin{array}{l}43 \cdot 00 \\
(16 \cdot 74-110 \cdot 50)\end{array}$ & - & $\begin{array}{l}6 \cdot 60 \\
(3 \cdot 27-13 \cdot 32)\end{array}$ & $\begin{array}{l}0 \cdot 07 \\
(0 \cdot 01-0 \cdot 31)\end{array}$ & $\begin{array}{l}0 \cdot 07 \\
(0 \cdot 036-0 \cdot 17)\end{array}$ & $\begin{array}{l}17 \cdot 6 \\
(7 \cdot 76-39 \cdot 7)\end{array}$ & $\begin{array}{l}0 \cdot 43 \\
(0 \cdot 18-0 \cdot 06)\end{array}$ & - \\
\hline & $P$ value & $0 \cdot 8151$ & 0.0302 & $<0.001$ & - & $<0.001$ & $<0.001$ & $<0.001$ & $<0.001$ & 0.0670 & - \\
\hline \multirow[t]{2}{*}{ Emba-Rufael* } & OR $(95 \% \mathrm{CI})$ & $\begin{array}{l}1 \cdot 00 \\
(0 \cdot 45-2 \cdot 22)\end{array}$ & $\begin{array}{l}0 \cdot 33 \\
(0 \cdot 15-0 \cdot 70)\end{array}$ & $\begin{array}{l}39 \cdot 93 \\
(15 \cdot 26-104 \cdot 49)\end{array}$ & - & $\begin{array}{l}4 \cdot 40 \\
(2 \cdot 19-8 \cdot 83)\end{array}$ & $\begin{array}{l}0 \cdot 12 \\
(0 \cdot 02-0 \cdot 60)\end{array}$ & $\begin{array}{l}0 \cdot 48 \\
(0 \cdot 21-1 \cdot 06)\end{array}$ & $\begin{array}{l}2 \cdot 35 \\
(1 \cdot 02-5 \cdot 42)\end{array}$ & $\begin{array}{l}1 \cdot 54 \\
(0 \cdot 49-4 \cdot 86)\end{array}$ & - \\
\hline & $P$ value & 0.9956 & 0.0039 & $<0.001$ & - & $<0.001$ & 0.0092 & 0.0714 & 0.0448 & 0.4557 & - \\
\hline
\end{tabular}

A, Mannheimia haemolytica serotype A; MA, Pasteurella multocida serotype A; T, Bibersteinia trehalosi serotype T; OR, odds ratio, CI, confidence interval; Ref., reference category.

* Univariable analysis where sheep and Lemlem peasant association were references. 
Table 2. M. haemolytica, P. multocida and B. trehalosi serotype prevalence in different age, bodyweight and sex as risk factors in sheep and goats in some locations of Tigray region

\begin{tabular}{|c|c|c|c|c|c|c|c|c|c|c|}
\hline & Summary statistics & $\mathrm{A} 1$ & $\mathrm{~A} 2$ & A7 & Total A & MA & $\mathrm{T} 3$ & $\mathrm{~T} 4$ & $\mathrm{~T} 10$ & $\mathrm{~T} 15$ \\
\hline \multicolumn{11}{|l|}{ Age } \\
\hline$<1$ yr $(n=19)$ & Positive (\%) & $10(66 \cdot 7)$ & $11(73 \cdot 3)$ & $7(46 \cdot 7)$ & & $7(46 \cdot 0)$ & $15(78 \cdot 9)$ & $11(73 \cdot 8)$ & $5(33 \cdot 3)$ & $11(73 \cdot 3)$ \\
\hline $1-2 \frac{1}{2}$ yr $(n=113)$ & Positive (\%) & $70(77 \cdot 8)$ & $67(74 \cdot 4)$ & $56(62 \cdot 2)$ & & $62(68 \cdot 9)$ & $100(88 \cdot 5)$ & $64(71 \cdot 1)$ & $38(42 \cdot 2)$ & $83(92 \cdot 2)$ \\
\hline$>2 \frac{1}{2} \mathrm{yr}(n=252)$ & Positive (\%) & $225(80 \cdot 6)$ & $205(73 \cdot 5)$ & $188(67 \cdot 6)$ & & $188(67 \cdot 4)$ & $209(82 \cdot 9)$ & $165(59 \cdot 1)$ & $116(41 \cdot 6)$ & $250(89 \cdot 6)$ \\
\hline$<1 \mathrm{yr}$ & Ref. & Ref. & Ref. & Ref. & & Ref. & Ref. & Ref. & Ref. & Ref. \\
\hline \multirow[t]{2}{*}{$1-2^{1 / 2} \mathrm{yr}$} & OR $(95 \% \mathrm{CI})$ & $\begin{array}{l}2 \cdot 69 \\
(0 \cdot 97-7 \cdot 45)\end{array}$ & $\begin{array}{l}0 \cdot 76 \\
(0 \cdot 25-2 \cdot 28)\end{array}$ & $\begin{array}{l}0 \cdot 90 \\
(0 \cdot 30-2 \cdot 71)\end{array}$ & & $\begin{array}{l}1 \cdot 62 \\
(0 \cdot 56-4 \cdot 38)\end{array}$ & $\begin{array}{l}2 \cdot 05 \\
(0 \cdot 59-7 \cdot 12)\end{array}$ & $\begin{array}{l}0 \cdot 78 \\
(0 \cdot 28-2 \cdot 15)\end{array}$ & $\begin{array}{l}1 \cdot 51 \\
(0 \cdot 55-4 \cdot 12)\end{array}$ & $\begin{array}{l}1 \cdot 88 \\
(0 \cdot 54-6 \cdot 49)\end{array}$ \\
\hline & $P$ value & $0 \cdot 0557$ & 0.6298 & 0.8573 & & $0 \cdot 3411$ & $0 \cdot 2580$ & $0 \cdot 6453$ & $0 \cdot 4173$ & $0 \cdot 3148$ \\
\hline \multirow[t]{2}{*}{$>21 / 2 \mathrm{yr}$} & OR $(95 \% \mathrm{CI})$ & $\begin{array}{l}3 \cdot 17(1 \cdot 20- \\
8 \cdot 32)\end{array}$ & $\begin{array}{l}1 \cdot 14 \\
(0 \cdot 39-3 \cdot 30)\end{array}$ & $\begin{array}{l}0 \cdot 58 \\
(0 \cdot 20-1 \cdot 66)\end{array}$ & & $\begin{array}{l}1 \cdot 45 \\
(0 \cdot 56-3 \cdot 75)\end{array}$ & $\begin{array}{l}1 \cdot 29 \\
(0 \cdot 41-4 \cdot 09)\end{array}$ & $\begin{array}{l}1 \cdot 06 \\
(0 \cdot 40-2 \cdot 81)\end{array}$ & $\begin{array}{l}1 \cdot 10 \\
(0 \cdot 42-2 \cdot 91)\end{array}$ & $\begin{array}{l}2 \cdot 78 \\
(0 \cdot 85-9 \cdot 13)\end{array}$ \\
\hline & $P$ value & $0 \cdot 0190$ & $0 \cdot 8052$ & $0 \cdot 3108$ & & 0.4384 & 0.6586 & 0.8934 & $0 \cdot 8334$ & 0.0903 \\
\hline \multicolumn{11}{|l|}{ Weight } \\
\hline$<0 \mathrm{~kg}(n=15)$ & Positive (\%) & $10(66 \cdot 7)$ & $11(73 \cdot 3)$ & $7(46 \cdot 7)$ & & $7(46 \cdot 0)$ & $15(78 \cdot 9)$ & $11(73 \cdot 8)$ & $5(33 \cdot 3)$ & $11(73 \cdot 3)$ \\
\hline $11-20 \mathrm{~kg}(n=90)$ & Positive (\%) & $70(77 \cdot 8)$ & $67(74 \cdot 4)$ & $56(62 \cdot 2)$ & & $62(68 \cdot 9)$ & $100(88 \cdot 5)$ & $64(71 \cdot 1)$ & $38(42 \cdot 2)$ & $83(92 \cdot 2)$ \\
\hline$>20 \mathrm{~kg}(n=279)$ & Positive (\%) & $225(80 \cdot 6)$ & $205(73 \cdot 5)$ & $188(67 \cdot 6)$ & & $188(67 \cdot 4)$ & $209(82 \cdot 9)$ & $165(59 \cdot 1)$ & $116(41 \cdot 6)$ & $250(89 \cdot 6)$ \\
\hline$<10 \mathrm{~kg}$ & Ref. & Ref. & Ref. & Ref. & & Ref. & Ref. & Ref. & Ref. & Ref. \\
\hline \multirow[t]{2}{*}{$11-20 \mathrm{~kg}$} & OR $(95 \% \mathrm{CI})$ & $\begin{array}{l}1 \cdot 75 \\
(0 \cdot 53-5 \cdot 71)\end{array}$ & $\begin{array}{l}1 \cdot 05 \\
(0 \cdot 30-3 \cdot 65)\end{array}$ & $\begin{array}{l}1 \cdot 88 \\
(0 \cdot 62-6 \cdot 71)\end{array}$ & & $\begin{array}{l}2 \cdot 53 \\
\quad(0 \cdot 83-6 \cdot 71)\end{array}$ & $\begin{array}{l}2 \cdot 25 \\
\quad(0 \cdot 53-9 \cdot 50)\end{array}$ & $\begin{array}{l}0 \cdot 89 \\
\quad(0 \cdot 26-3 \cdot 06)\end{array}$ & $\begin{array}{l}1 \cdot 46 \\
(0 \cdot 47-4 \cdot 27)\end{array}$ & $\begin{array}{l}4 \cdot 31 \\
\quad(1 \cdot 08-17 \cdot 13)\end{array}$ \\
\hline & $P$ value & $0 \cdot 3537$ & 0.9274 & $0 \cdot 2598$ & & $0 \cdot 1006$ & $0 \cdot 2698$ & $0 \cdot 8600$ & 0.5186 & 0.0379 \\
\hline \multirow[t]{2}{*}{$>20 \mathrm{~kg}$} & OR $(95 \% \mathrm{CI})$ & $\begin{array}{l}2 \cdot 08 \\
(0 \cdot 68-6 \cdot 34)\end{array}$ & $\begin{array}{l}1 \cdot 01 \\
(0 \cdot 31-3 \cdot 26)\end{array}$ & $\begin{array}{l}2 \cdot 36 \\
(0 \cdot 83-6 \cdot 71)\end{array}$ & & $\begin{array}{l}2 \cdot 36 \\
(0 \cdot 83-6 \cdot 71)\end{array}$ & $\begin{array}{l}1 \cdot 20 \\
(0 \cdot 32-4 \cdot 42)\end{array}$ & $\begin{array}{l}0 \cdot 52 \\
(0 \cdot 16-1 \cdot 69)\end{array}$ & $\begin{array}{l}1 \cdot 42 \\
(0 \cdot 47-4 \cdot 27)\end{array}$ & $\begin{array}{l}3 \cdot 13 \\
(0 \cdot 93-10 \cdot 48)\end{array}$ \\
\hline & $P$ value & $0 \cdot 3537$ & 0.9902 & $0 \cdot 1070$ & & $0 \cdot 1070$ & 0.7809 & $0 \cdot 2818$ & 0.5292 & $0 \cdot 0636$ \\
\hline \multicolumn{11}{|l|}{ Sex } \\
\hline Male $(n=77)$ & Positive (\%) & $62(80 \cdot 5)$ & $50(64 \cdot 9)$ & $53(68 \cdot 8)$ & $76(98 \cdot 7)$ & $55(71 \cdot 4)$ & $66(85 \cdot 7)$ & $45(58 \cdot 4)$ & $30(39 \cdot 0)$ & $69(89 \cdot 6)$ \\
\hline Female $(n=307)$ & Positive (\%) & $243(79 \cdot 2)$ & $233(75 \cdot 9)$ & $198(64 \cdot 7)$ & $300(97 \cdot 7)$ & $202(65 \cdot 8)$ & $258(84 \cdot 0)$ & $195(63 \cdot 5)$ & $129(42 \cdot 0)$ & $275(89 \cdot 6)$ \\
\hline Male & Ref. & Ref. & Ref. & Ref. & Ref. & Ref. & Ref. & Ref. & Ref. & Ref. \\
\hline \multirow[t]{2}{*}{ Female } & OR $(95 \% \mathrm{CI})$ & $\begin{array}{l}0 \cdot 91 \\
(0 \cdot 49-1 \cdot 72)\end{array}$ & $\begin{array}{l}1 \cdot 70 \\
(0 \cdot 99-2 \cdot 90)\end{array}$ & $\begin{array}{l}0 \cdot 83 \\
(0 \cdot 48-1 \cdot 41)\end{array}$ & $\begin{array}{l}0 \cdot 56 \\
(0 \cdot 06-4 \cdot 63)\end{array}$ & $\begin{array}{l}0 \cdot 76 \\
(0 \cdot 44-1 \cdot 33)\end{array}$ & $\begin{array}{l}0 \cdot 87 \\
(0 \cdot 43-1 \cdot 78)\end{array}$ & $\begin{array}{l}1 \cdot 23 \\
(0 \cdot 74-2 \cdot 06)\end{array}$ & $\begin{array}{l}1 \cdot 13 \\
(0 \cdot 68-1 \cdot 89)\end{array}$ & $\begin{array}{l}0 \cdot 99 \\
(0 \cdot 43-2 \cdot 25)\end{array}$ \\
\hline & $P$ value & 0.7909 & $0 \cdot 0523$ & $0 \cdot 4963$ & $0 \cdot 594$ & $0 \cdot 3486$ & $0 \cdot 7175$ & $0 \cdot 4112$ & $0 \cdot 6262$ & 0.9931 \\
\hline
\end{tabular}

A, Mannheimia haemolytica serotype A; MA, Pasteurella multocida serotype A; T, Bibersteinia trehalosi serotype T; OR, odds ratio, CI, confidence interval; Ref., reference category. 


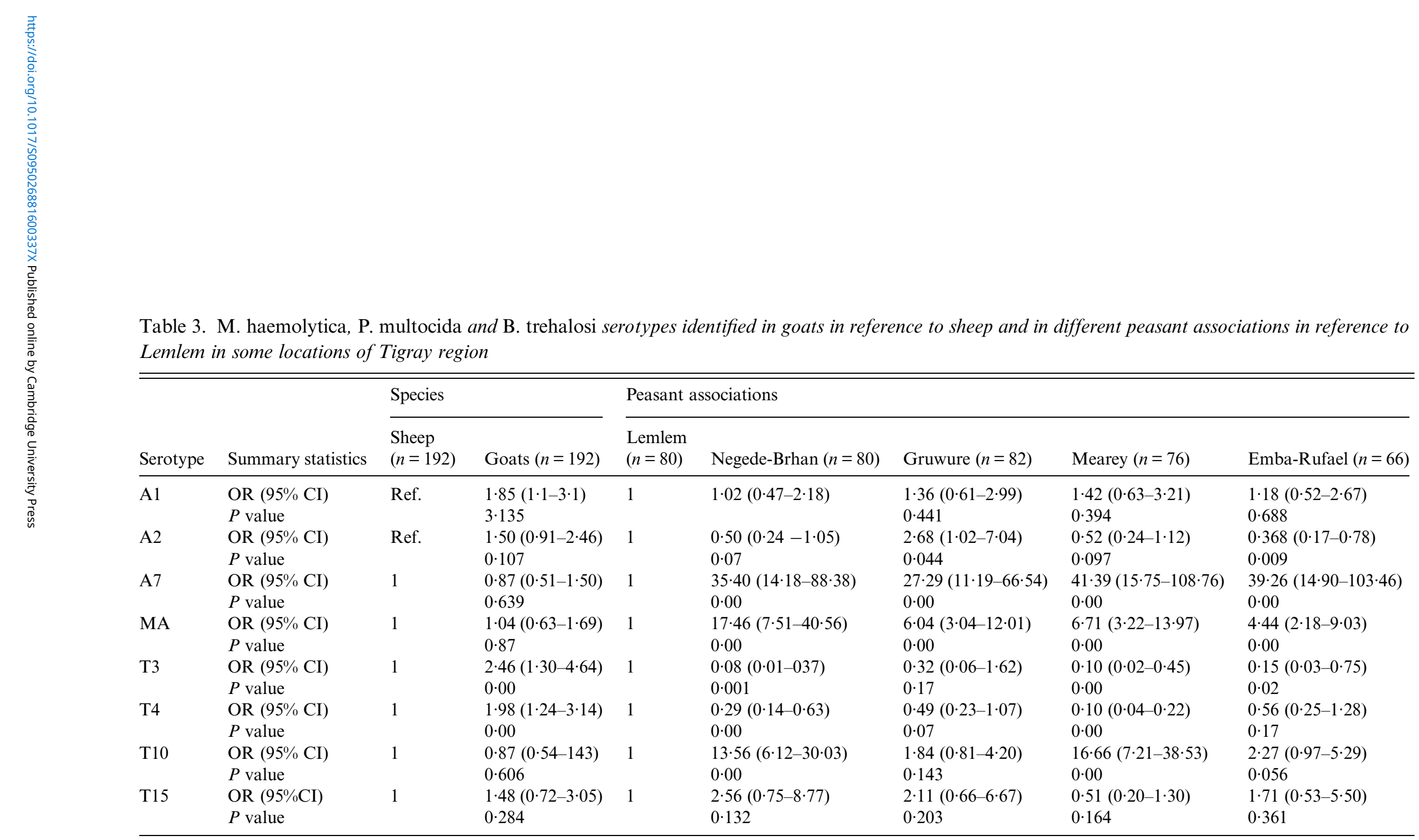

A, Mannhaemia haemolytica serotype A; MA, Pasteurella multocida serotype A; T, Bibersteinia trehalosi serotype T; OR, odds ratio, CI, confidence interval; Ref., reference category. 
Table 4. The distribution of total summarized combination patterns of mixed serotype co-infection per single animal among small ruminants $(n=384)$ of the studied areas in Tigray region

\begin{tabular}{llllllllll}
\hline \hline & \multicolumn{9}{l}{ No. of serotypes infecting single animal } \\
\cline { 2 - 10 } & 1 & 2 & 3 & 4 & 5 & 6 & 7 & 8 & Total \\
\hline No. of various patterns for serotype combinations & 3 & 6 & 13 & 25 & 28 & 20 & 7 & 1 & 103 \\
No. of infected animal & 4 & 6 & 19 & 46 & 91 & 97 & 87 & 34 & 384 \\
\% of infected animals & $1 \cdot 04$ & $1 \cdot 56$ & 4.95 & $11 \cdot 98$ & $23 \cdot 70$ & $25 \cdot 26$ & $22 \cdot 66$ & $8 \cdot 85$ \\
\hline \hline
\end{tabular}

Overall, eight serotypes were detected. No single negative animal was observed among all of the examined animals $(n=384)$ as each animal was infected by at least one or more serotypes. The eight serotypes displayed a total of 103 different (co)-infection patterns across the 384 infected animals using different serotype combinations. For example, 25.26\% (97/ 384 ) of the animals were infected by six serotypes with 20 different combination (cluster) patterns.

distribution, risk factors for positivity to different serotypes, and potential within-animal clustering of seropositivity were assessed in Pasteurella, Mannheimia and Bibersteinia in sheep and goats in selected areas of Tigray region.

Overall, $98 \cdot 7 \%$ prevalence was found for both $M$. haemolytica and $B$. trehalosi (any serotype) and $66.9 \%$ for $P$. multocida serotype A in this study, indicating the former two are particularly widespread. At the individual serotype level, the prevalence was highest for the T15, T3 and A1 serotypes. A previous report from Northern Ireland similarly reported that B. trehalosi was more common than M. haemolytica in sheep [31]. However, other studies have reported that $M$. haemolytica is the most frequent serotype in sheep and goats in Egypt [32] and in northwestern Ethiopia [30]. In the current study, all sampled animals were seropositive to at least one serotype. This could be due to the commensal presence of some of these organisms as normal constituents of the nasal and pharyngeal microflora of healthy ruminants [3335]. Healthy small ruminants are frequent carriers of these commensal opportunistic bacteria genera which tend to impose significant health and economic problems mainly when animals are under stressful conditions [34, 35]. However, the high prevalence and multiple circulating serotypes in the area could cause substantial animal health and economic impacts, as the sub-optimal animal management by smallholder farmers would exacerbate the impact of pasteurellosis.

The current study evaluated 384 sera for eight serotypes using the IHA test. All eight tested serotypes were frequently identified among sheep and goats: A1 (79.4\%), A2 (73.7\%), A7 (65.5\%), MA (66.9\%), T3 $(84 \cdot 4 \%)$, T4 $(62 \cdot 5 \%), \quad \mathrm{T} 10(41 \cdot 4 \%)$ and $\mathrm{T} 15$ (89.6\%). A complication of pasteurellosis control is that known serotypes do not produce cross-immunity
[4]. Complex clonal diversity of strains has been observed within $M$. haemolytica serotypes [36] particularly within A1 and A2 [37] and within B. trehalosi $\mathrm{T} 4$ and T15 serotypes [38] in which each unique clone appears to have host-specific tropism [9] and can act as a primary pathogen, causing disease outbreaks in the specific host species. In this study the most common serotypes in goats were serotypes A1, A2, T3 and T4 and in sheep A7 and T10. Even after controlling for the variation between different PAs, goats had higher odds of T3 and T4 infection compared to sheep. In a similar study in Hungary, only serotype A2 was frequently detected in goats, and the remaining serotypes were very rare, while all serotypes were detected in sheep except A14 [39]. Variation in the detection of serotypes between sheep and goats could be explained by differing host tropism between serotypes [9]. In this study P. multocida serotype A was another important infection which was detected in sheep with prevalence slightly higher than in goats. P. multocida is emerging as a common pneumonic pasteurellosis disease in sheep and goats [40, 41 , it is more commonly encountered in tropical and subtropical regions [41].

Variations in the age, sex and body condition of the animals did not appear to impact the seroprevalence of the majority of the studied serotypes. However, serotypes A1 and T15 were more prevalent in adult age $(>2.5$ years) and in slightly heavier bodyweight (11$20 \mathrm{~kg}$ ) small ruminants, respectively. It is not known if serotypes $\mathrm{A} 1$ and $\mathrm{T} 15$ have unique tropism to animals of specific age groups or bodyweight, although host-specific tropism has been reported for a number of serotypes and for subgroups within a serotype [9, $36,38,42,43]$.

One limitation of serosurveys is their inability to differentiate past exposure from recent infection. 
Table 5. Distribution of the detailed serotype combination patterns $(n=103$ patterns) for co-infection in small ruminants $(n=384)$ from five locations of Tigray region

\begin{tabular}{|c|c|c|c|c|c|c|c|}
\hline $\begin{array}{l}\text { Serotype combination pattern } \\
\text { for co-infection }\end{array}$ & $\begin{array}{l}\text { No. of infected } \\
\text { animals of } 384 \\
\text { examined }\end{array}$ & $\begin{array}{l}\% \text { of infected } \\
\text { animals of } \\
384\end{array}$ & $\begin{array}{l}\text { No. of serotypes } \\
\text { involved in the } \\
\text { pattern, out of } 8\end{array}$ & $\begin{array}{l}\text { Serotype combination } \\
\text { pattern for co-infection }\end{array}$ & $\begin{array}{l}\text { No. of infected } \\
\text { animals of } 384 \\
\text { examined }\end{array}$ & $\begin{array}{l}\% \text { of infected } \\
\text { animals of } \\
384\end{array}$ & $\begin{array}{l}\text { No. of serotypes } \\
\text { involved in the } \\
\text { pattern, out of } 8\end{array}$ \\
\hline A1A2A7MAT3T4T15 & 43 & $11 \cdot 20$ & 7 & A1A2T3T4T10T15 & 1 & $0 \cdot 26$ & 6 \\
\hline A1A2A7MAT3T4T10T15 & 34 & $8 \cdot 85$ & 8 & A1A2A7MAT4T10 & 1 & $0 \cdot 26$ & 6 \\
\hline A1A2T3T4T15 & 30 & $7 \cdot 81$ & 5 & A1A2A7MAT3T4 & 1 & $0 \cdot 26$ & 6 \\
\hline A1A2A7T3T4T15 & 22 & $5 \cdot 73$ & 6 & A2PAT3T4T15 & 1 & $0 \cdot 26$ & 5 \\
\hline A1A2A7MAT3T10T15 & 19 & 4.95 & 7 & A2A7T4T10T15 & 1 & $0 \cdot 26$ & 5 \\
\hline A1A2MAT3T4T15 & 13 & $3 \cdot 39$ & 6 & A2A7MAT3T4 & 1 & $0 \cdot 26$ & 5 \\
\hline A1A2A7MAT10T15 & 12 & $3 \cdot 13$ & 6 & A2A7MAT3T15 & 1 & $0 \cdot 26$ & 5 \\
\hline A1A2MAT3Т4T10T15 & 10 & $2 \cdot 60$ & 7 & A2A7MAT3T10 & 1 & $0 \cdot 26$ & 5 \\
\hline A1MAT3T4T15 & 9 & $2 \cdot 34$ & 5 & A1A7T3T4T10 & 1 & $0 \cdot 26$ & 5 \\
\hline A1A7MAT3Т4T15 & 8 & $2 \cdot 08$ & 6 & A1A7MAT4T15 & 1 & $0 \cdot 26$ & 5 \\
\hline A1A7MAT3T4T10T15 & 6 & $1 \cdot 56$ & 7 & A1A7MAT4T10 & 1 & $0 \cdot 26$ & 5 \\
\hline A1A2A7MAT3T15 & 6 & $1 \cdot 56$ & 6 & A1A7MAT3T15 & 1 & $0 \cdot 26$ & 5 \\
\hline A2A7MAT3Т4T15 & 5 & $1 \cdot 30$ & 6 & A1A2MAT3T10 & 1 & $0 \cdot 26$ & 5 \\
\hline A2A7MAT3Т10T15 & 5 & $1 \cdot 30$ & 6 & A1A2MAT3T10 & 1 & $0 \cdot 26$ & 5 \\
\hline A1A7T3T4T15 & 5 & $1 \cdot 30$ & 5 & A1A2A7T3T4 & 1 & $0 \cdot 26$ & 5 \\
\hline A1A2MAT3T15 & 5 & $1 \cdot 30$ & 5 & A1A2A7T3T4 & 1 & $0 \cdot 26$ & 5 \\
\hline A1MAT3T15 & 5 & $1 \cdot 30$ & 4 & A1A2A7T10T15 & 1 & $0 \cdot 26$ & 5 \\
\hline A1A2T3T15 & 5 & $1 \cdot 30$ & 4 & A1A2A7MAT3 & 1 & $0 \cdot 26$ & 5 \\
\hline A2A7MAT3T4T10T15 & 4 & $1 \cdot 04$ & 7 & T3T4T10T15 & 1 & $0 \cdot 26$ & 4 \\
\hline A1A2A7MAT4T10T15 & 4 & $1 \cdot 04$ & 7 & PAT3T4T15 & 1 & $0 \cdot 26$ & 4 \\
\hline A1A2MAT3T10T15 & 4 & $1 \cdot 04$ & 6 & A7MAT3T15 & 1 & $0 \cdot 26$ & 4 \\
\hline A1A2A7MAT3T10 & 4 & $1 \cdot 04$ & 6 & A7MAT10T15 & 1 & $0 \cdot 26$ & 4 \\
\hline A7MAT3T10T15 & 4 & $1 \cdot 04$ & 5 & A2MAT3T4 & 1 & $0 \cdot 26$ & 4 \\
\hline A2A7T3T4T15 & 4 & $1 \cdot 04$ & 5 & A2MAT3T15 & 1 & $0 \cdot 26$ & 4 \\
\hline A1A7MAT10T15 & 4 & $1 \cdot 04$ & 5 & A2MAT10T15 & 1 & $0 \cdot 26$ & 4 \\
\hline A7MAT4T15 & 4 & $1 \cdot 04$ & 4 & A2A7MAT10 & 1 & $0 \cdot 26$ & 4 \\
\hline A1T3T15 & 4 & $1 \cdot 04$ & 3 & A1A7T4T15 & 1 & $0 \cdot 26$ & 4 \\
\hline A1A7MAT4T10T15 & 3 & 0.78 & 6 & A1A7T3T15 & 1 & $0 \cdot 26$ & 4 \\
\hline A1A7MAT3T10T15 & 3 & $0 \cdot 78$ & 6 & A1A7T3T10 & 1 & $0 \cdot 26$ & 4 \\
\hline A1A2A7T3T10T15 & 3 & $0 \cdot 78$ & 6 & A1A7MAT10 & 1 & $0 \cdot 26$ & 4 \\
\hline А2MAT3Т10T15 & 3 & $0 \cdot 78$ & 5 & A1A2T3T10 & 1 & $0 \cdot 26$ & 4 \\
\hline A1MAT3T10T15 & 3 & $0 \cdot 78$ & 5 & A1A2MAT3 & 1 & $0 \cdot 26$ & 4 \\
\hline A1A2A7T3T15 & 3 & $0 \cdot 78$ & 5 & $\mathrm{~A} 1 \mathrm{~A} 2 \mathrm{~A} 7 \mathrm{MA}$ & 1 & $0 \cdot 26$ & 4 \\
\hline A7MAT10T15 & 3 & $0 \cdot 78$ & 4 & T3T4T15 & 1 & $0 \cdot 26$ & 3 \\
\hline A2T3T4T15 & 3 & $0 \cdot 78$ & 4 & T3T4T15 & 1 & $0 \cdot 26$ & 3 \\
\hline A1T3T4T15 & 3 & $0 \cdot 78$ & 4 & MAT3T15 & 1 & $0 \cdot 26$ & 3 \\
\hline
\end{tabular}




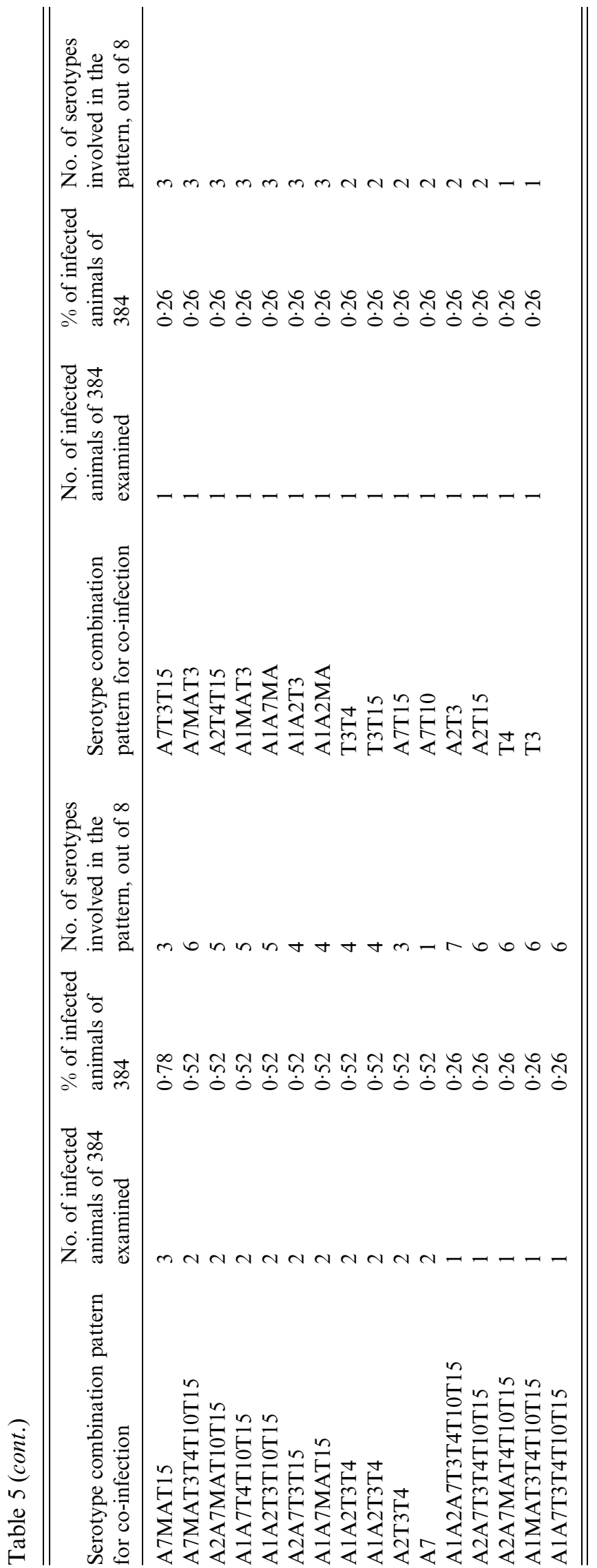

Thus, the current study could not discriminate whether the higher seroprevalence in the adult age groups was a reflection of the long-term cumulative accumulation of antibodies due to past exposure or due to recent or current infection. Generally, however, $M$. haemolytica causes septicaemia in young lambs and pneumonia in all ages while $B$. trehalosi causes acute systemic disease affecting principally the upper alimentary tract and lungs in young adults [44].

Each locality (PA) had a different magnitude of prevalence for each of the eight serotypes studied and only PA explained a significant proportion of the variation (adjusted $R^{2}=0 \cdot 16$ ) in the serological responses. Variation in seroprevalence between PAs may be due to geographical variation in type and level of predisposing factors, serotype abundance and/or the immune status of the studied animals [29-32]. While the most prevalent Pasteurella strain varied between PAs, even within PAs, there was considerable variation in the serotypes to which each animal had been exposed, with the two major clusters appearing to be A7, MA and T10; or T3 and T4. The distribution of these five serotypes through the population of the studied small ruminants of different locations (PAs) points towards contagious spread while the remaining three serotypes seem a totally endogenous infection with limited evidence of spread within the wider flock. Similar findings have been reported previously [42, 43].

The clustering of certain serotypes of Pasteurella within animals may reflect common pathways of exposure or differing genetic or acquired susceptibility in certain groups of animals. While not significant in RDA, multivariable logistic regression suggested that T3 and T4 were more likely to be found in goats than sheep; however, the results from the RDA suggest that, even in sheep, these strains are more likely to be found together than would be expected if the two strains were independent. After the effect of PA was accounted for, B. trehalosi strains T3 and T4 were observed to be positively correlated within animals: however, T4 strain was observed to have negative correlations with the T10 strain, suggesting they were less likely to be found within the same animal than might be expected under an assumption of independence. M. haemolytica strain A7 was clustered with $P$. multocida serotype A, and to a lesser extent with $B$. trehalosi strain T10 within animals.

The implications for vaccine production of these findings are that, due to the considerable variation 

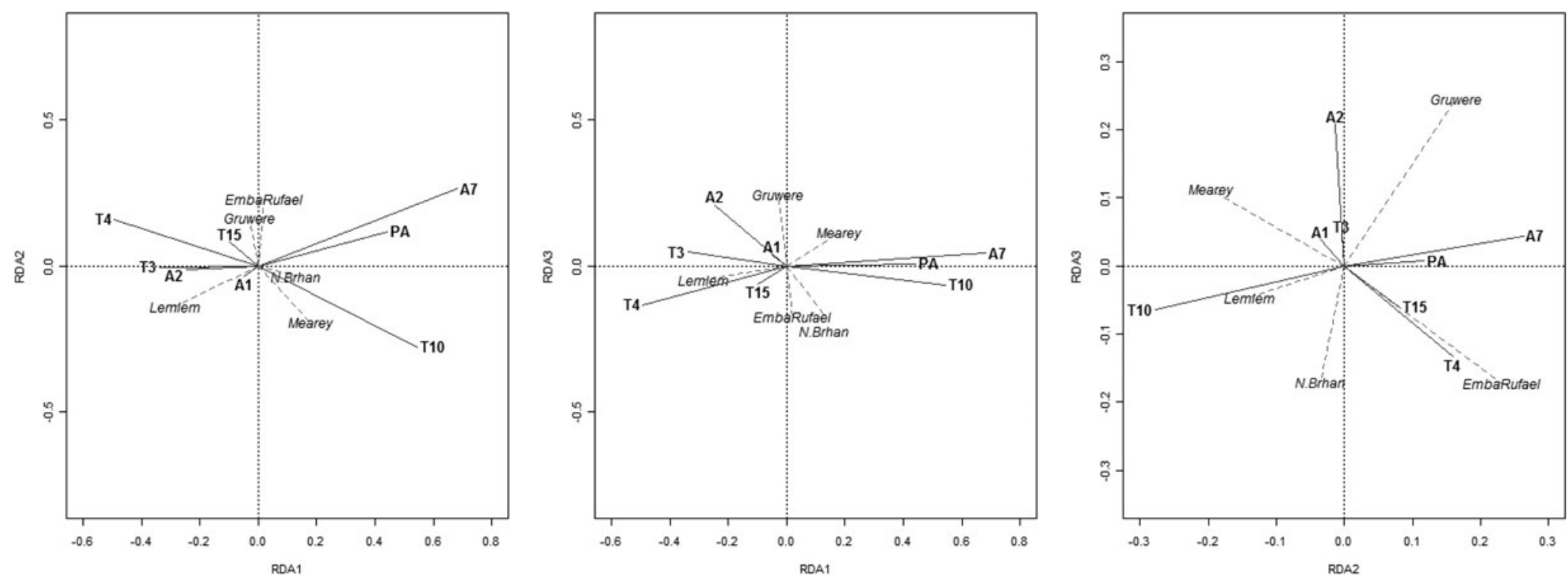

Fig. 1. Mannheimia, Pasteurella and Bibersteinia serotype co-infections analysed for potential of clustering in sheep and goats in different peasant associations (PAs) Triplots of the three significant redundancy analysis (RDA) axes, showing relationships between explanatory and response variables. Solid lines represent serological responses, dashed lines represent PAs. Angles between variables represent their correlations. 
in strain exposure both between PAs and between animals in the same PA, it is difficult to select serotypes for a polyvalent vaccine based on serology alone. However, as many Pasteurella infections may be clinically silent, or only be significant as secondary infections in the presence of other pathogens, combination of this serological data with data from clinical case presentations and pathogen isolation may provide valuable information in selecting the serotypes of greatest importance in this population. Importantly, the significant variation between PAs may suggest caution should be exercised when extending these findings to other areas.

\section{CONCLUSION}

The current serological study revealed the widespread distribution of eight serotypes of pasteurellosis agents among localities, sheep and goats in Tigray region. Of the risk factors evaluated, age (serotype A1) and bodyweight (serotype T15) were significantly associated with infection, but sex was not significant. Serotypes A1, A2, T3 and T4 were significantly associated with goats; A7 and T10 with sheep; P. multocida serotypes A and T15 with both sheep and goats; A1 and T15 with all the five PAs while serotypes A2, A7, T3, T4, and T10 were associated with at least one of the five PAs. Only PA explained a significant proportion of the variation (adjusted $R^{2}=0 \cdot 16$ ) in the serological responses. After the effect of PA was accounted for, T3 and T4 were positively correlated with co-infection while $\mathrm{T} 4$ and T10 had negative correlations and were less likely to be found within the same animal. Serotype A7 was clustered with $P$. multocida serotype A, and to a lesser extent T10 within animals. Overall $355(92 \cdot 45 \%)$ animals were infected with four or more serotype combinations with potential of clustering serotype per animal. The diversity of serotypes observed here indicates that currently available vaccines are likely to be unable adequately to protect against pasteurellosis. The results of this study clearly indicate the need for further investigation to better understand the epidemiology of pasteurellosis across a wider geographical area, including investigation of the clonal diversity of pasteurellosis pathogens responsible for clinical cases, in order to inform recommendations for the selection of serotypes for inclusion in polyvalent vaccines.

\section{ACKNOWLEDGEMENTS}

The financial support of Addis Ababa University College of Veterinary Medicine and Agriculture
Postgraduate Programme as well as partial financial support from Mekelle Regional Veterinary Diagnostic and Research Laboratory to Kassaye Berhe is greatly appreciated.

\section{DECLARATION OF INTEREST}

None.

\section{REFERENCES}

1. Heddleston KL, Gallagher JE, Rebers PA. Fowl cholera: gel diffusion precipitin test for serotyping Pasteurella multocida from avian species. Avian Diseases 1972; 16: 925-936.

2. Carter GR. Studies on Pasteurella multocida. I. A hemagglutination test for the identification of serological types. American Journal of Veterinary Research 1955; 16: $481-484$.

3. Biberstein EL, Gills M, Knight H. Serological types of Pasteurella hemolytica. Cornell Veterinarian 1960; 50: 283-300.

4. Fodor L, Penzes Z, Varga J. Coagglutination test for serotyping Pasteurella haemolytica. Journal of Clinical Microbiology 1996; 34: 393-397.

5. Angen $\mathbf{O}$, et al. Taxonomic relationships of the [Pasteurella] haemolytica complex as evaluated by DNA-DNA hybridizations and 16S rRNA sequencing with proposal of Mannheimia haemolytica gen. nov., comb. nov., Mannheimia granulomatis comb. nov., Mannheimia glucosida sp. nov., Mannheimia ruminalis sp. nov. and Mannheimia varigena sp. nov. International Journal of Systematic Bacteriology 1999; 49: 67-86.

6. Villard L, et al. Serological and molecular comparison of Mannheimia haemolytica and Pasteurella trehalosi strains isolated from wild and domestic ruminants in the French Alps. Veterinary Journal 2006; 171: 545-550.

7. Odugbo MO, et al. The comparative pathogenicity of strains of eight serovars and untypable strains of Mannheimia haemolytica in experimental pneumonia of sheep. Veterinary Research 2004; 35: 661-669.

8. Purdy CW, Cooley JD, Straus DC. Cross-protection studies with three serotypes of Pasteurella haemolytica in the goat model. Current Microbiology 1998; 36: 207-211.

9. Harper M, Boyce JD, Adler B. Pasteurella multocida pathogenesis: 125 years after Pasteur. FEMS Microbiology Letters 2006; 265:1-10.

10. Foreyt WJ, Jessup DA. Fatal pneumonia of bighorn sheep following association with domestic sheep. Journal of Wildlife Diseases 1982; 18: 163-168.

11. Callan RJ, et al. Development of pneumonia in desert bighorn sheep after exposure to a flock of exotic wild and domestic sheep. Journal of the American Veterinary Medical Association 1991; 198: 1052-1056.

12. Kehrenberg $\mathbf{C}$, et al. Antimicrobial resistance in Pasteurella and Mannheimia: epidemiology and genetic basis. Veterinary Research 2001; 32: 323-339. 
13. Verma RS, Jaiswa TN. Haemorrhagic septicaemia vaccines. Vaccine 1998; 16: 1184-1192.

14. Ferede Y, et al. Serotyping and evaluation of the level of protective antibody titer in Northwest Ethiopian sheep before and after ovine pasteurollosis vaccination. International Journal of Pharma Medicine and Biological Science 2013; 2: 57-64.

15. Deressa A, et al. Molecular detection of Pasteurella multocida and Mannheimia haemolytica in sheep respiratory infections in Ethiopia. Journal of Applied Research in Veterinary Medicine 2010; 8: 101-108.

16. Pegram RG, Roeder PL, Scott JM. Two new serotypes of Pasteurella haemolytica from sheep in Ethiopia. Tropical Animal Health and Production 1979; 11: 29-30.

17. Tibbo M, Woldemeskel M, Gopilo A. An outbreak of respiratory disease complex in sheep in central Ethiopia. Tropical Animal Health and Production 2001; 33: 355-365.

18. Yimer N, Asseged B. Aerobic bacterial flora of the respiratory tract of healthy sheep slaughtered in Dessie municipal abattoir, northeastern Ethiopia. Revue de Médécine Vétérinaire 2007; 158: 473-478.

19. Marru HD, Anijajo TT, Hassen AA. A study on Ovine pneumonic pasteurellosis: Isolation and Identification of Pasteurellae and their antibiogram susceptibility pattern in Haramaya District, Eastern Hararghe, Ethiopia. BMC Veterinary Research 2013; 9: 239.

20. Desta D, et al. Analysis of goat value chains in Tanqua Abergelle district, Tigray, Ethiopia. International Livestock Research Institute, 2013, pp. 45 (https:// cgspace.cgiar.org/handle/10568/35378).

21. Ebrahim A, Hailemichael A. Sheep and goat production and utilization in different agro-ecological zones in Tigray, Ethiopia. Livestock Research for Rural Development 2012; 24 (http://www.lrrd.cipav.org.co/ lrrd24/1/asse24016.htm).

22. Hassen $\mathbf{H}$, et al. Molecular characterization of Ethiopian indigenous goat populations. Tropical Animal Health and Production 2012; 44: 1239-1246.

23. Berhane G, Eik LO. Effect of vetch (Vicia sativa) hay supplementation on performance of Begait and Abergelle goats in northern Ethiopia: I. Milk yield and composition. Small Ruminant Research 2006; 64: 225-232.

24. Thrusfield MV, Veterinary Epidemiology, 3rd edn. New Jersey: Wiley, 2007, pp.

25. Biberstein EL, Thompson DA. Epidemiological studies on Pasteurella haemolytica in sheep. Journal of Comparative Pathology 1966; 76:83-94.

26. Frank GH, Wessman GE. Rapid plate agglutination procedure for serotyping Pasteurella haemolytica. Journal of Clinical Microbiology 1978; 7: 142-145.

27. Oksanen JF, et al. Vegan: Community Ecology Package, 2011. R package version 1.17-11 (http:// CRAN.R-project.org/package $=$ vegan).

28. Borcard D, Gillet F, Legendre P. Introduction. In: Numerical Ecology with R. New York: Springer, 2011, pp. 1-7.
29. Mohamed RA, Abdelsalam EB. A review on pneumonic pasteurellosis (respiratory mannheimiosis) with emphasis on pathogenesis, virulence mechanisms and predisposing factors. Bulgarian Journal of Veterinary Medicine 2008; 11: 139-160.

30. Engdaw TA, Alemneh AT. Pasteurellosis in small ruminants: biochemical isolation, characterization and prevalence determination in relation to associated risk factors in Fogera Woreda, North-West Ethiopia. Advances in Biological Research 2015; 9: 330-337.

31. Ball HJ, Connolly M, Cassidy J. Pasteurella haemolytica serotypes isolated in Northern Ireland during 1989-1991. British Veterinary Journal 1993; 149: 561-570.

32. Kaoud H, et al. Occurrence of Mannheimia haemolytica and Pasteurella trehalosi among ruminants in Egypt. New York Science Journal 2010; 3: 135-141.

33. Richard Y, et al. Nasal and pulmonary flora in the goat. Annals of Veterinary Research 1988; 20: 269-276.

34. Jasni S, et al. Isolation of Pasteurella haemolytica from the nasal cavity of goats. British Veterinary Journal 1991; 147: 352-355.

35. Brogden KA, Lehmkuhl HD, Cutlip RC. Pasteurella haemolytica complicated respiratory infections in sheep and goats. Veterinary Research 1998; 29: 233-254.

36. Davies RL, Donachie W. Intra-specific diversity and host specificity within Pasteurella haemolytica based on variation of capsular polysaccharide, lipopolysaccharide and outer-membrane proteins. Microbiology 1996; 142: 1895-1907.

37. Villard L, et al. Serotypes A1 and A2 of Mannheimia haemolytica are susceptible to genotypic, capsular and phenotypic variations in contrast to T3 and T4 serotypes of Bibersteinia (Pasteurella) trehalosi. FEMS Microbiology Letters 2008; 280: 42-49.

38. Davies RL, Quirie M. Intra-specific diversity within Pasteurella trehalosi based on variation of capsular polysaccharide, lipopolysaccharide and outermembrane proteins. Microbiology 1996; 142: 551-560.

39. Fodor L, et al. Serotypes of Pasteurella haemolytica and Pasteurella trehalosi isolated from farm animals in Hungary. Journal of Veterinary Medicine, Series B 1999; 46: 241-247.

40. Zamri-Saad M, et al. The goat as a model for studies of pneumonic pasteurellosis caused by Pasteurella multocida. British Veterinary Journal 1996; 152: 453-458.

41. Odugbo MO, et al. Pasteurella multocida pneumonic infection in sheep: prevalence, clinical and pathological studies. Small Ruminant Research 2006; 66: 273-277.

42. Shreeve BJ, Biberstein EL, Thompson DA. Variation in carrier rates of Pasteurella haemolytica in sheep: II. Diseased flocks. Journal of Comparative Pathology 1972; 82: 111-116.

43. Shreeve BJ, Thompson DA. Studies on the carriage of Pasteurella haemolytica in lambs. Journal of Comparative Pathology 1970; 80:107-112.

44. Gilmour NJL. Pasteurella haemolytica infections in sheep. Veterinary Quarterly 1980; 2: 191-198. 